\title{
Relationship of Renal Echogenicity with Renal Pathology and Function
}

\author{
Jin Hee Lee, M.D. ${ }^{1}$ \\ Myung Hyun Cho, M.D. ${ }^{1}$ \\ Sung III Chung, M.D. ${ }^{2}$ \\ So Dug Lim, M.D. ${ }^{3}$ \\ Kyo Sun Kim, M.D. ${ }^{1}$
}

Departments of Pediatrics ${ }^{1}$, Radiology ${ }^{2}$, Pathology ${ }^{3}$, Konkuk University Medical Center, Seoul, Republic of Korea

Corresponding author: Kyo Sun Kim, M.D. Department of Pediatrics, Konkuk University Medical Center, Konkuk University School of Medicine, 120-1 Neungdong-ro, Gwangjin-gu, Seoul 05030, Korea

Tel: $+82-2-2030-7379$

Fax: +82-2-2030- 7748

E-mail:kimkyo@kuh.ac.kr

Received: 24 July 2017

Revised: 21 August 2017

Accepted: 18 September 2017
Purpose: Renal ultrasonography has been widely used in children with renal disease. However, the relationship of renal echogenicity with renal pathology and function in children is not well known.

Method: Ultrasound examination was performed in 75 patients undergoing renal biopsy for suspected renal disease in Konkuk University Medical Center from August 2005 to November 2015. We compared renal echogenicity to pathologic findings and renal function. Renal echogenicity was scored as 0 to 2 by comparing adjacent liver echogenicity. Three histologic characteristics were evaluated: glomerular changes, interstitial infiltration or fibrosis, and tubular atrophy. These were graded as 0 to 3 , according to increasing severity. Laboratory results included urine albumin excretion and estimated glomerular filtration rate (eGFR).

Results: Among pathologic findings, renal echogenicity revealed a positive correlation with interstitial infiltration or fibrosis $(\mathrm{r}=0.259, P=0.025)$, and with tubular atrophy $(\mathrm{r}=0.268, P=0.02)$. Renal echogenicity and glomerular changes were not correlated . Renal echogenicity showed a positive correlation with microalbuminuria $(\mathrm{r}=0.283, P=0.014)$, but a negative correlation with eGFR $(\mathrm{r}=-0.352, P=0.002)$. Conclusion: Increased renal echogenicity suggested severe interstitial infiltration or fibrosis and tubular atrophy among the pathologic findings. Moreover, increased echogenicity is correlated with increased urine albumin excretion and decreased eGFR. Echogenicity on ultrasonography is useful for determining the status of renal pathology and function.

Key words: Renal echogenicity, Renal pathology, Albuminuria, Glomerular Filtration Rate, Children

\section{Introduction}

Kidneys had four contents; the glomerulus, the tubules, the interstitium and the vessels. Depending on the type of renal diseases, one content was changed or all contents were changed. However, all contents were changed as diseases were progressed, so at the end stage, it was difficult to know the origin of all content $^{1 \text { ) }}$. If a biopsy was done before the disease progresses, we could diagnose the disease and know where the damage part is. However, a biopsy was invasive, so it was performed only when indication was matched.

Ultrasonography has been widely used to evaluate the condition of the kidneys in patients with renal diseases ${ }^{2}$. Sonography provides the information about length, contour, and the relative echogenicity and pattern of the cortex
This is an open-access article distributed under the terms of the Creative Commons Attribution Non-Commercial License (http:// creativecommons.org/licenses/by-nc/4.0/) permits unrestricted non-commercia medium, provided the original work is properly cited.

Pediatric Nephrology 
and medulla ${ }^{3)}$. Sonography shows not only anatomic information but also the boundary between tissue organization and it suggests the pathologic condition of an organ ${ }^{4}$. Moreover, sonography is noninvasive and does not use ionizing radiation ${ }^{5}$. In children, it does not require sedation. Therefore, it is useful to evaluate the condition of kidneys and diagnose renal diseases, especially in children.

Increased renal parenchymal echogenicity would be used as a predictor of decreased renal function ${ }^{6}$. However, renal ultrasound results could be changed according to the tester. To get over this limitation, the kidney compared with the liver or the spleen, and close relationships between renal echogenicity and renal pathology or laboratory exam were reported $^{3,7,8)}$. Nevertheless, it is not enough to use as a diagnostic discriminator alone, so sometimes renal biopsy is needed to diagnose the renal diseases more specifically. At present, the condition of kidneys is reflected through renal biopsy, but it is invasive and it would lead to complications such as anuria, inflammation and bleeding. If we can predict the condition of kidney by ultrasonography, it will be helpful to diagnose and treat the patient.

We aimed to evaluate the relationship between renal echogenicity and histopathologic changes in children with renal disease. Furthermore, we also explored relationship between renal echogenicity and renal function.

\section{Materials and methods}

We retrospectively studied children who had renal sonography and renal biopsy in Konkuk University Medical Center from August 2005 to November 2015 to correlate renal echogenicity and renal histopathology. Indication for kidney biopsy was the patient with steroid resistant nephrotic syndrome, persistent proteinuria and hematuria over 6 months, acute renal failure, and decreased renal function. We included children between 3 and 18 years old. They did not receive treatment before the examination except two nephrotic syndrome children. They had steroid treatment. Children who had renal sonography after renal biopsy were excluded. Patients who had hepatic disease based on clinical and laboratory findings were excluded. Out of 86 patents who had both examinations, 75 patients met our criteria, and were reviewed retrospectively. Also, technically ade- quate sonograms and adequate biopsy specimens were evaluated independently.

All sonographic examinations were obtained with a 1.5 or $5.8 \mathrm{MHz}$ convex transducer by one radiologist. Gains were altered to optimized imaging by a radiologist. Renal echogenicity was graded from 0 to 2 by comparing renal echogenicity to the adjacent liver echogenicity; grade 0 : the renal echogenicity was less than that of the liver; grade 1 : the renal echogenicity equaled that of the liver; grade 2 : the renal echogenicity was greater than that of the liver (Fig. 1). One radiologist classified the grade of renal echogenicity without the knowledge of clinical, laboratory findings and biopsy results.

In all patients, sonographically guided renal biopsies were gained from the left kidney using a TSK acecut biopsy needle by the operator. Two pathologists evaluated the pathologic findings and classified the results. Three histologic characteristics were evaluated: glomerular alteration, interstitial infiltration or fibrosis and tubular atrophy, which were measured from 0 to 3 dependent on the increasing severity (Table 2). Vascular alterations were not considered because those in all pathologic findings were not specific. Glomerular alteration was graded from 0 to 3; grade 0: no change, grade 1: minimal change, grade $2:<50 \%$ glomerular obsolescence, grade 3: > 50\% glomerular obsolescence. Interstitial infiltration or fibrosis was graded from 0 to 3 ; grade 0: no change, grade 1: minimal cellular infiltration or fibrosis, grade 2: moderate cellular infiltration or fibrosis, grade 3: marked or severe cellular infiltration or fibrosis. Tubular atrophy was graded from 0 to 3 ; grade 0 : no change, grade 1: minimal, grade 2: focal atrophy, grade 3: marked or severe atrophy.

The laboratory examinations included serum creatinine level, urine albumin creatinine ratio ( $\mathrm{mg} / \mathrm{g}$ ), and urinalysis. The estimated glomerular filtration rate (eGFR) was calculated based on bedside Schwartz formula: $0.413 \times$ height $(\mathrm{cm}) /$ serum creatinine $(\mathrm{mg} / \mathrm{g})^{9}$. . Random voided urine was collected for determination of albuminuria and hematuria. Albuminuria was graded from 0 to 2; grade 0 : normal defined as a urinary albumin excretion lower than $30 \mathrm{mg} / \mathrm{g}$ creatinine, grade 1: microalbuminuria defined as a urinary albumin excretion of 30-300 mg/g creatinine, grade 2: macroalbuminuria defined as a urinary albumin excretion higher than $300 \mathrm{mg} / \mathrm{g}$ creatinine. Hematuria was graded 
from 0 to 2; grade 0: 0 to 4 red blood cells per high-power field of urine sediment, grade 1: 5 to 49 red blood cells per high-power field of urine sediment, grade 2: 50 and over red blood cells per high-power field of urine sediment. Renal echogenicity was correlated with the estimated severity of disease (microalbumin in urine, blood in urine), and pathologic criteria.

Data are expressed as numbers (percentages) for categorical variables, and as mean for continuous variables. SPSS software, version 23.0 was used. Spearman correlation coefficients were used to examine relationships between grade of renal echogenicity and grade of renal pathology, albuminuria, hematuria. Pearson correlation analyses were performed to analyze the eGFR for grade of renal echogenicity. Fisher's exact test was used to analyze relationship between renal echogenicity and eGFR of the normal group and the abnormal group. $P$-values $<0.05$ and $<0.1$ were considered statistically significant.

\section{Results}

Baseline characteristics are shown in Table 1. Total 75 patients were enrolled in this study. They were 3 years to 18 years old (mean age 12.0 years of age). Mean eGFR was $91.99 \mathrm{~mL} / \mathrm{min} / 1.73 \mathrm{~m}^{2}$. There were 39 males and 36 females. All patients had abnormal laboratory findings and clinical symptoms about renal diseases. They had no hepatic diseases based on clinical and laboratory findings. Renal echogenicity was considered grade 0 in 33 patients, grade 1 in 38 patients, and grade 2 in 4 patients. Albuminuria was considered grade 0 in 44 patients, grade 1 in 28 patients, and grade 2 in 3 patients. Hematuria was considered grade 0 in 23 patients, grade 1 in 35 patients, and grade 2 in 17 patients (Table 1).

The renal diseases of patients were IgA nephropathy ( $\mathrm{N}=$ 16), thin basement membrane disease $(\mathrm{N}=14)$, mesangial proliferative glomerulonephritis $(\mathrm{N}=8)$, acute postinfectious glomerulonephritis $(\mathrm{N}=4)$, focal segmental glomerulosclerosis $(\mathrm{N}=2)$, membranous glomerulonephritis $(\mathrm{N}=2)$, membranoproliferative glomerulonephritis $(\mathrm{N}=2)$, HenochScheonlein Purpura nephritis $(\mathrm{N}=2)$, acute interstitial nephritis $(\mathrm{N}=2)$, lupus nephritis $(\mathrm{N}=1)$, mild focal nonspecific glomerulonephritis $(\mathrm{N}=1)$, thickening of glomerular capil- lary basement membrane $(\mathrm{N}=1)$, tubular cell injury $(\mathrm{N}=1)$, and minor change $(\mathrm{N}=19)$. Patients with minor change also had renal biopsy because they had proteinuria or microscopic hematuria more than 6 months.

Data of three histologic parameters (glomerular alteration, interstitial infiltration or fibrosis, and tubular atrophy) was presented in Table 2. In glomerular alteration, 33 patients showed grade 0 in renal echogenicity. Among them, 48.4\% patients showed glomerular alteration although they have no change of echogenicity. Among 38 patients in grade

Table 1. Characteristics of 75 Pediatric Patients in the Study Group

\begin{tabular}{lc}
\hline & Number (\%), or mean \\
\hline Gender (male:female) & $39: 36$ \\
Age at evaluation (years) & $11.61 \pm 4.01$ \\
eGFR $\left(\mathrm{mL} / \mathrm{min}^{2} / .73 \mathrm{~m}^{2}\right)$ & $91.99 \pm 31.62$ \\
Kidney echogenicity & \\
Grade 0 & $33(44)$ \\
Grade 1 & $38(51)$ \\
Grade 2 & $4(5)$ \\
Albuminuria & \\
Grade 0 & $44(59)$ \\
Grade 1 & $28(37)$ \\
Grade 2 & $3(4)$ \\
Hematuria & \\
Grade 0 & $23(30)$ \\
Grade 1 & $35(47)$ \\
Grade 2 & $17(23)$ \\
\hline
\end{tabular}

Table 2. Distribution of Grade of Renal Echogenicity and Histopathologic Parameters

\begin{tabular}{lccl}
\hline Group & \multicolumn{3}{c}{ Grade of echogenicity } \\
\hline Pathology findings & $0(\mathrm{n}=33)$ & $1(\mathrm{n}=38)$ & $2(\mathrm{n}=4)$ \\
\hline Glomerular alteration & & & \\
Grade 0 & $17(51.6 \%)$ & $19(50.0 \%)$ & $0(0 \%)$ \\
Grade 1 & $8(24.2 \%)$ & $11(28.9 \%)$ & $2(50.0 \%)$ \\
Grade 2 & $8(24.2 \%)$ & $8(21.1 \%)$ & $1(25.0 \%)$ \\
Grade 3 & $0(0 \%)$ & $0(0 \%)$ & $1(25.0 \%)$ \\
Interstitial infiltration or fibrosis & & & \\
Grade 0 & $27(81.8 \%)$ & $25(65.8 \%)$ & $1(25.0 \%)$ \\
Grade 1 & $5(15.1 \%)$ & $12(31.6 \%)$ & $2(50.0 \%)$ \\
Grade 2 & $1(3.1 \%)$ & $1(2.6 \%)$ & $1(25.0 \%)$ \\
Grade 3 & $0(0 \%)$ & $0(0 \%)$ & $0(0 \%)$ \\
Tubular atrophy & & & \\
Grade 0 & $27(81.8 \%)$ & $25(65.8 \%)$ & $1(25.0 \%)$ \\
Grade 1 & $2(6.1 \%)$ & $4(10.5 \%)$ & $0(0 \%)$ \\
Grade 2 & $4(12.1 \%)$ & $9(23.7 \%)$ & $3(75.0 \%)$ \\
Grade 3 & $0(0 \%)$ & $0(0 \%)$ & $0(0 \%)$ \\
\hline
\end{tabular}


1 of renal echogenicity, $28.9 \%$ patients had grade 1 of glomerular alteration and $21.1 \%$ patients had grade 2 of glomerular alteration, but $50 \%$ patients had no change histopathologically. Also, 4 patients had grade 2 of renal echogenicity and all patients had glomerular change. In interstitial infiltration or fibrosis, most patients of grade 0 in renal echogenicity had no change histopathologically. Also, patients of grade 1 in renal echogenicity were 38 and $34.2 \%$ patients had grade 1 or grade 2 of interstitial infiltration or fibrosis. There were 4 patients with grade 2 in renal echogenicity, and 75\% patients had interstitial infiltration or fibrosis. In tubular atrophy, $18.2 \%$ patients of grade 0 and $34.2 \%$ patients of grade 1 in renal echogenicity revealed tubular abnormality. Patients of grade 2 in renal echogenicity were 4 and $75 \%$ patients showed tubular atrophy in pathologic findings (Table 2).

Renal echogenicity showed a positive correlation with interstitial infiltration or fibrosis $(\mathrm{r}=0.259, P=0.025)$, and tubular atrophy $(\mathrm{r}=0.268, P=0.020)$. There was no significant relationship between renal echogenicity and glomerular alteration (Table 3).

A negative correlation was found between renal echogenicity and eGFR $(P=0.002)$. A positive correlation was found between renal echogenicity and albuminuria $(P=$ $0.014)$. No correlation was found between hematuria and renal echogenicity (Table 3).

Also, we examined the relationship between renal echogenicity and eGFR of the normal group and the abnormal group. Normal eGFR has been known to be 79-187 mL/ $\mathrm{min} / 1.73 \mathrm{~m}^{2}$ in $2-12$ years old children and $82-170 \mathrm{~mL} / \mathrm{min} /$ $1.73 \mathrm{~m}^{2}$ in $13-21$ years old children ${ }^{10)}$. $P$-value was 0.005 , so renal echogenicity was associated with eGFR of the normal group and the abnormal group.

Table 3. Correlations between Grade of Renal Echogenicity and Renal Function or Pathologic Findings

\begin{tabular}{lcc}
\hline & Coefficient & $P$-value \\
\hline Albuminuria & $0.283^{*}$ & 0.014 \\
Hematuria & 0.111 & 0.341 \\
eGFR & $-0.352^{\dagger}$ & 0.002 \\
Pathology & & \\
Glomerular & 0.104 & 0.373 \\
Interstitial & $0.259^{*}$ & 0.025 \\
Tubular & $0.268^{*}$ & 0.020 \\
\hline
\end{tabular}

${ }^{*} P<0.05,{ }^{\dagger} P<0.01$.
Most common diseases in this study were IgA nephropathy $(\mathrm{N}=16)$, thin basement membrane disease $(\mathrm{N}=14)$, and mesangial proliferative glomerulonephritis $(\mathrm{N}=8)$. Among these groups, we evaluated relationship of renal echogenicity with renal pathology and renal function respectively, and no correlation was found.

We examined relationship of renal echogenicity with renal pathology and renal function in whole patients except patients with minor change. Renal echogenicity showed a positive correlation with glomerular alteration $(\mathrm{r}=0.265$, $P=0.049)$, interstitial infiltration or fibrosis ( $\mathrm{r}=0.260, P=$ $0.053)$, and tubular atrophy $(\mathrm{r}=0.263, P=0.050)$. A negative correlation was found between renal echogenicity and eGFR ( $\mathrm{r}=-0.311, P=0.020)$. There was a positive correlation between renal echogenicity and albuminuria $(\mathrm{r}=0.338, P=$ 0.011 ). As in the previous results, there was no correlation between hematuria and renal echogenicity.

In the case of glomerular diseases, the components of the glomerular occupied about $8 \%$ of the renal parenchyma and there might be no significant change in the renal echogenicity. However, as the disease progresses, histopathologic parenchymal elements changed, so renal echogenicity became increased. In the tubulointerstitial diseases, echogenicity of medulla was increased. In nephrotic syndrome, there was no change, or parenchymal echogenicity was increased.

A sensitivity of $58 \%, 72 \%, 72 \%$ and a specificity of $52 \%$, $50 \%, 50 \%$ for finding glomerular alteration, interstitial infiltration or fibrosis, Tubular atrophy, retrospectively, have been reported in renal echogenicity.

\section{Discussion}

Ultrasonography is the most commonly used imaging tool in kidney disease. We evaluated the relationship of sonographic findings, especially echogenicity with pathologic parameters and renal function. There were a positive correlation of interstitial infiltration or fibrosis and tubular atrophy with echogenicity. Also, kidney echogenicity had the negative correlation with eGFR and the positive correlation with albuminuria.

Renal cortex was hypoechogenic as comparing to the liver or the spleen in healthy children and adults ${ }^{11)}$. The 
mechanism of increased renal echogenicity was induced by increased perfusion because of the change of cell infiltration and fibrous remodeling ${ }^{12)}$. Therefore, isoechogenic renal echogenicity with liver or spleen echogenicity would suggest renal disease ${ }^{12)}$.

There were several studies to evaluate the status of the kidney through echogenicity ${ }^{3,78}$. However, the results were inconsistent. Hricak et al. ${ }^{8)}$ observed that echogenicity was positively correlated with histologically glomerular changes. Meanwhile, the other studies reported the positive correlation of increased echogenicity with interstitial changes. However, there was no definite relation between echogenicity and glomerular lesions ${ }^{7,13}$. From Moghazi et al. ${ }^{14)}$ renal echogenicity was related to severity of tubular atrophy and interstitial inflammation. Brenbridge et al. ${ }^{3)}$ suggested that renal echogenicity correlated with interstitial infiltration, glomerular disease and tubular atrophy in children. In this study, we know that there was no correlation between glomerular changes on biopsy and the sonographic results. However, there was correlation between interstitial changes and tubular atrophy on biopsy and the echogenicity. This matched that the anatomy of the cortex formed with interstitial tissue and tubules mostly ${ }^{7}$. However, in the patients excluding patients with minor change, glomerular alteration was correlated with renal echogenicity. As the disease progresses, the kidney tissues changed among glomerulus, interstitium and vessels, so renal echogenicity was increased $^{2}$.

Some studies demonstrated that increased renal parenchymal echogenicity would be used as a predictor of decreased renal function ${ }^{6}$. The increased right kidney-liver echogenicity ratio showed a close relationship with decreased GFR in children ${ }^{15}$. There was important correlation between cortical echogenicity and blood urea nitrogen and creatinine concentrations ${ }^{8}$. In this study, there was negative correlation between renal echogenicity and eGFR.

There were few studies about relationship between renal sonography, pathology and function in children. In this study, we evaluated children among 3 years to 18 years old. Moreover, the findings of renal sonography were evaluated with not only the results of renal pathology but also eGFR, urine albumin excretion and hematuria. So it is meaningful in pediatric nephrology.

This study had several limitations. First, hydration of pa- tients was not controlled. Renal echogenicity was effected by hydration in children ${ }^{16)}$. Han and Babcock ${ }^{12)}$ reported that renal cortex was isoechogenic from neonates to 6 months of age. Therefore, from 7 months isoechogenic renal echogenicity would suggest renal parenchymal disease $^{12)}$. However, after hydration, renal echogenicity could be increased because renal tubules were expended ${ }^{17,18)}$. Therefore, standardization of hydration would lead to better result before renal ultrasonography. Second, the number of participated children was small. Furthermore, most children show normal or minimal change in the test of sonography and biopsy.

This is the report about correlation of renal echogenicity with renal histopathology, eGFR, albumin in urine, and hematuria in pediatric patients. Interstitial infiltration or fibrosis and tubular atrophy are correlated with renal echogenicity. Also, high urine albumin excretion, low eGFR were related to renal echogenicity. Based on this study, if you look at the ultrasound of patients with pediatric renal diseases, you can predict the histological changes of interstitum and tubule, and infer the function of the kidneys. More studies are needed to predict parenchymal disease by sonographic discriminator.

\section{Conflicts of interest}

No potential conflict of interest relevant to this article was reported.

\section{References}

1. Buturovic-Ponikvar J, Visnar-Perovic A. Ultrasonography in chronic renal failure. Eur J Radiol 2003;46(2):115-22.

2. Quaia E, Bertolotto M. Renal parenchymal diseases: is characterization feasible with ultrasound? Eur Radiol 2002;12(8):2006-20.

3. Brenbridge AN, Chevalier RL, Kaiser DL. Increased renal cortical echogenicity in pediatric renal disease: histopathologic correlations. J Clin Ultrasound 1986;14(8):595-600.

4. O'Neill WC. Sonographic evaluation of renal failure. Am J Kidney Dis 2000;35(6):1021-38.

5. Lucisano G, Comi N, Pelagi E, Cianfrone P, Fuiano L, Fuiano G. Can renal sonography be a reliable diagnostic tool in the assessment of chronic kidney disease? J Ultrasound Med 2015;34(2):299-306. 
6. Chi T, Feldstein VA, Nguyen HT. Increased echogenicity as a predictor of poor renal function in children with grade 3 to 4 hydronephrosis. J Urol 2006;175(5):1898-901.

7. Rosenfield AT, Siegel NJ. Renal parenchymal disease: histopathologic-sonographic correlation. AJR Am J Roentgenol 1981;137(4): 793-8.

8. Hricak H, Cruz C, Romanski R, Uniewski MH, Levin NW, Madrazo $B L$, et al. Renal parenchymal disease: sonographic-histologic correlation. Radiology 1982;144(1):141-7.

9. Schwartz GJ, Munoz A, Schneider MF, Mak RH, Kaskel F, Warady $B A$, et al. New equations to estimate GFR in children with CKD. J Am Soc Nephrol 2009;20(3):629-37.

10. Ghasemi A, Azimzadeh I, Afghan M, Momenan AA, Bagheripour F, Azizi F. Pediatric reference values for serum creatinine and estimated glomerular filtration rate in Iranians: Tehran Lipid and Glucose Study. Arch Iran Med 2015;18(11):753-9.

11. Krensky AM, Reddish JM, Teele RL. Causes of increased renal echogenicity in pediatric patients. Pediatrics 1983;72(6):840-6.

12. Han BK, Babcock DS. Sonographic measurements and appearance of normal kidneys in children. AJR Am J Roentgenol 1985; 145(3):611-6.
13. Page JE, Morgan SH, Eastwood JB, Smith SA, Webb DJ, Dilly SA, et al. Ultrasound findings in renal parenchymal disease: comparison with histological appearances. Clin Radiol 1994;49(12): 867-70.

14. Moghazi S, Jones E, Schroepple J, Arya K, McClellan W, Hennigar RA, et al. Correlation of renal histopathology with sonographic findings. Kidney Int 2005;67(4):1515-20.

15. Lee YS, Lee MJ, Kim MJ, Im YJ, Kim SW, Lim NL, et al. Is Increased Echogenicity Related to a Decrease in Glomerular Filtration Rate? Objective Measurements in Pediatric Solitary Kidney Patients--A Retrospective Analysis. PLoS One 2015;10(8):e0133577.

16. Peerboccus M, Damry N, Pather S, Devriendt A, Avni F. The impact of hydration on renal measurements and on cortical echogenicity in children. Pediatr Radiol 2013;43(12):1557-65.

17. Manley JA, O'Neill WC. How echogenic is echogenic? Quantitative acoustics of the renal cortex. Am J Kidney Dis 2001;37(4):70611.

18. Vehmas T, Kaukiainen A. Factors associated with renal cortical echogenicity. Ultrasound Med Biol 2006;32(8):1151-5. 